\title{
The head shapes of some mammalian spermatozoa and their possible relationship to the shape of the penetration slit through the zona pellucida
}

\author{
D. P. L. Green \\ Department of Pharmacology, University of Otago Medical School, P.O. Box 913, Dunedin, \\ New Zealand
}

\begin{abstract}
Summary. A method has been developed for establishing from electron micrographs the radius of curvature of rabbit and guinea-pig sperm nuclei in head-to-tail section (the major axis), and of guinea-pig spermatozoa with intact acrosomes in transverse section (the minor axis). Radii of curvature of head-to-tail sections lie, for the most part, between 7 and $100 \mu \mathrm{m}$ in both species. Guinea-pig sperm nuclei in transverse section show a range of radii between 3 and $10 \mu \mathrm{m}$, and approximate, therefore, caps of prolate spheroids. The method is not applicable to rat and mouse spermatozoa but Nomarski light microscopy suggests that these nuclei are straight in the longitudinal direction. The distribution of the radii of curvature in rabbit sperm nuclei is similar to that of the radii of curvature of penetration slits through the zona pellucida of the rabbit egg. It is suggested that longitudinal curvature of sperm heads is correlated with any curvature of the penetration slit.
\end{abstract}

Keywords: mammalian spermatozoa; head shape; penetration slit; zona pellucida

\section{Introduction}

The mammalian egg at fertilization is surrounded by two concentric investments. The outermost, the corona radiata, is composed of residual follicle cells embedded in an extracellular matrix; the inner investment, the zona pellucida, is an amorphous matrix of glycoproteins (Bleil \& Wassarman, 1980; Dietl \& Czuppon, 1984; Greve \& Wassarman, 1985). During the course of fertilization, spermatozoa pass between the cells of the corona radiata and, in some species, attach, approximately tangentially, to the zona pellucida. Those that do attach tangentially then move through the zona pellucida in an arcuate path to the perivitelline space (Dickmann, 1964; Dickmann \& Dziuk, 1964; Dziuk \& Dickmann, 1965; Yanagimachi, 1966; Yang et al., 1972). In mouse and rat eggs, however, penetration is apparently normal to the zona surface and the penetration slit straight (Austin \& Braden, 1956; Gaddum-Rosse et al., 1984). The immediate cause of the curvature has never been identified although prima facie it plays an important part in ensuring that spermatozoa meet the egg in those species in which penetration of the zona pellucida is initially close to tangential. One possibility is that any curvature of the slit in the zona is caused by the curvature of the sperm head in longitudinal section and that, as a corollary, straight slits are a consequence of heads which are straight in longitudinal section.

In this paper, a procedure is described which uses electron micrographs to establish a radius of curvature for head-to-tail sections of rabbit and guinea-pig sperm nuclei. The method is not applicable to rat and mouse spermatozoa. All spermatozoa were sectioned optically by Nomarski light microscopy to give qualitative data. 


\section{Materials and Methods}

Glutaraldehyde (25\%) was purchased from Agar Aids (Bishop's Stortford, Herts, U.K.); ethylene glycol tetra-acetic acid (EGTA) and Hepes from Sigma (St Louis, MO, U.S.A.), and Ficoll 400 from Pharmacia (Uppsala, Sweden). All other reagents were analytical grade. A23187 was a generous gift of Dr R. L. Hamill (Eli Lilly Laboratories, Indianapolis, IN, U.S.A.). Gelatin was manufactured by Davis Gelatine (NZ) Limited (Christchurch, New Zealand).

The procedures for obtaining and handling epididymal spermatozoa were as previously described (Green, 1978). Spermatozoa with intact acrosomes were fixed after suspension in medium containing $140 \mathrm{~mm}-\mathrm{NaCl}, 4 \mathrm{~mm}-\mathrm{KCl}$, $4 \mathrm{~mm}$-Hepes, $10 \mathrm{~mm}$-glucose, $2 \mathrm{~mm}-\mathrm{MgCl}_{2}$, and $100 \mu \mathrm{M}$-EGTA. The acrosome reaction was induced in guinea-pig spermatozoa by addition of $10 \mu \mathrm{l}$ of a stock solution of $A 23187$ in dimethyl sulphoxide $(2 \mathrm{mg} / \mathrm{ml})$ to $1 \mathrm{ml}$ of spermatozoa suspended in a medium similar to that described above, but with the substitution of $2 \mathrm{mM}-\mathrm{CaCl}_{2}$ for the $2 \mathrm{mM}-\mathrm{MgCl}_{2}$ and $100 \mu \mathrm{M}$-EGTA. Spermatozoa were fixed $5 \mathrm{~min}$ after addition of the A23187. Sperm suspensions contained about $5 \times 10^{7}$ spermatozoa $/ \mathrm{ml}$.

Preparations for electron microscopy were as previously described (Green, 1978). Silver-to-gold sections were cut on a Sorvall microtome, post-stained with $1 \%$ uranyl acetate in $50 \%$ alcohol and Reynold's lead citrate, and examined in a Philips 300 electron microscope.

Spermatozoa were examined by Nomarski light microscopy on a Zeiss IM35 inverted microscope using a $\times 63$ oil immersion objective (N.A. 1.4).

A perspex model of a sperm head was constructed with the following dimensions: length, $23 \mathrm{~mm}$, maximum width, $13 \mathrm{~mm}$, maximum thickness at base, $1.5 \mathrm{~mm}$, thickness at tip, $0.7 \mathrm{~mm}$. A ridge $0.1 \mathrm{~mm}$ thick and $0.5 \mathrm{~mm}$ deep was left on the anterior half of the head to mimic the perforatorium. The maximum concavity of the head was approximately $0.6 \mathrm{~mm}$. The head itself was attached to a $70 \mathrm{~mm}$ length of wire (diameter, $1.5 \mathrm{~mm}$ ). If the length of the head is taken as being proportional to a sperm head length of $6 \mu \mathrm{m}$, the radius of curvature is, relatively speaking, approximately $30 \mu \mathrm{m}$, and the perforatorium $26 \mathrm{~nm}$ thick and $0.13 \mu \mathrm{m}$ deep. The model head was driven slowly into a $6 \%$ gelatin gel cast in a perspex chamber $(90 \mathrm{~mm} \times 90 \mathrm{~mm} \times 27 \mathrm{~mm})$ until it was completely immersed, and illuminated transversely with polarized, monochromatic light from a sodium lamp $(589$ and $589.6 \mathrm{~nm})$. It was viewed through a crossedpolarizer which was rotated to give zero light transmission under conditions of zero stress. The applied load was approximately $30-40 \mathrm{~g}$.

\section{Results}

Measurements were made to establish whether the nuclei of spermatozoa are curved in longitudinal section.

A pellet of spermatozoa prepared for electron microscopy has spermatozoa orientated at random throughout (with the exception of the stacks of guinea-pig spermatozoa in which each spermatozoon has the same orientation but individual stacks are orientated randomly). Guinea-pig and rabbit spermatozoa possess heads which are paddle-shaped, with bilateral symmetry about the major, i.e. head-to-tail, axis. The method for determining their nuclear curvature depends on recognizing sections which run along this axis. This is done by adopting the working rule that any section through the sperm head which lies on the axis must contain at least an equal length of tail. This working rule is based on two assumptions: (1) that the tail abuts the head centrally, and (2) that for the first few micrometres of tail, bending in the plane of the head is sufficiently restricted to ensure that the angle about which the section pivots, at the base of the head, is small. Both these assumptions need some qualification. Firstly, it has been recognized for some time that there is a distribution of the point of attachment of head and tail on either side of the major axes of guineapig sperm heads (Fawcett, 1968; Friend \& Fawcett, 1974). Little can be done to correct for this but, for reasons which become clear later, it does not affect the conclusion. Secondly, evidence from Nomarski light micrographs suggests that, in any section in which the length of the tail at least equals the length of the head, the angle of the section to the major axis, in the plane of the head, should be no more than about $7^{\circ}$ and in most cases much less (data not shown). This conclusion is supported by the micrographs in Friend \& Fawcett (1974). There is also a small but unquantified deflection of the tail out of the plane of the head in guinea-pig spermatozoa (Fawcett, 1965). It has no effect on the analysis other than ensuring that those sections actually measured are a population marginally closer to the perpendicular to the plane of the head than might otherwise have been the case. Whether this flexure applies to the spermatozoa of other species is unclear. 

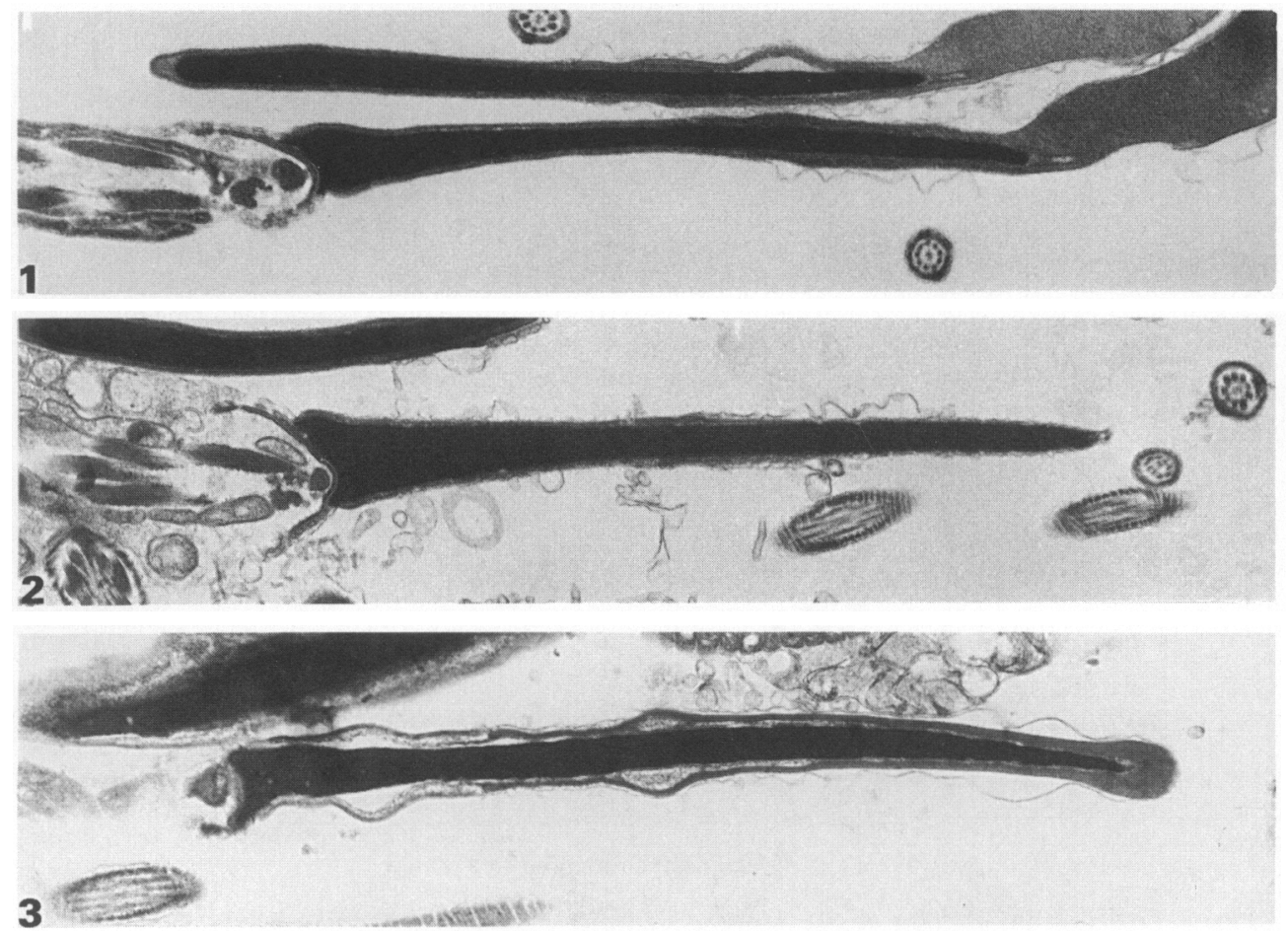

Fig. 1. Longitudinal sections through two guinea-pig sperm heads, both with intact acrosomes. Only one spermatozoon possesses an equal length of tail within the section and therefore satisfies the criteria laid down in the 'Results' section for measurement. The acrosome is on its convex face. $\times 22700$.

Fig. 2. As in Fig. 1, but without acrosome. $\times 22100$.

Fig. 3. Head-to-tail section through rabbit sperm head. $\times 22600$.

The procedure establishes sections which are on, or close to, the major axis of the head. These sections represent, however, all the sections which have the axis as a rotational axis. A consequence of this is that almost all sections will show some degree of obliquity to the perpendicular to the plane of the head surface. If the head itself was a flat plate, oblique sections through the head would be straight. Since sections through the head are seldom straight, it can be concluded that the heads of rabbit and guinea-pig spermatozoa are not wholly flat along the major axis. There is, however, considerable diversity in the shape, thickness, and curvature of sperm nuclei, all of which is difficult to quantify. Typical sections through the heads of guinea-pig and rabbit spermatozoa are shown in Figs 1-3.

The approach to quantification has been to relate each nuclear section to the arc of a hypothetical circle. To do this, a line is drawn between the mid-point at the anterior tip of the nucleus and that at the posterior region of the nucleus where it attaches to the tail (Fig. 4). This line is then treated as if it were the chord of a circle. Taking a perpendicular from its mid-point to the centre line of the nucleus gives the sagitta of a circle and hence a radius of curvature. The hypothetical circle so defined will coincide with a line down the centre of a nucleus in section if (a) the nuclear mid-line in head-to-tail section lies on the arc of a circle, and (b) this section is perpendicular to the plane of the head. The projection of this arc onto an oblique surface along the major axis would be a parabola and, for small angles of arc, a new circle could be drawn through the ends of the arc and the apex of the parabola, in effect, generating a new and larger sagitta. As the angle of projection 


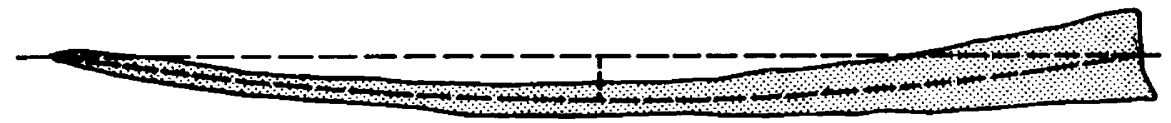

Fig. 4. Diagrammatic view of a guinea-pig sperm nucleus indicating the chord used in the calculation of the hypothetical radius.

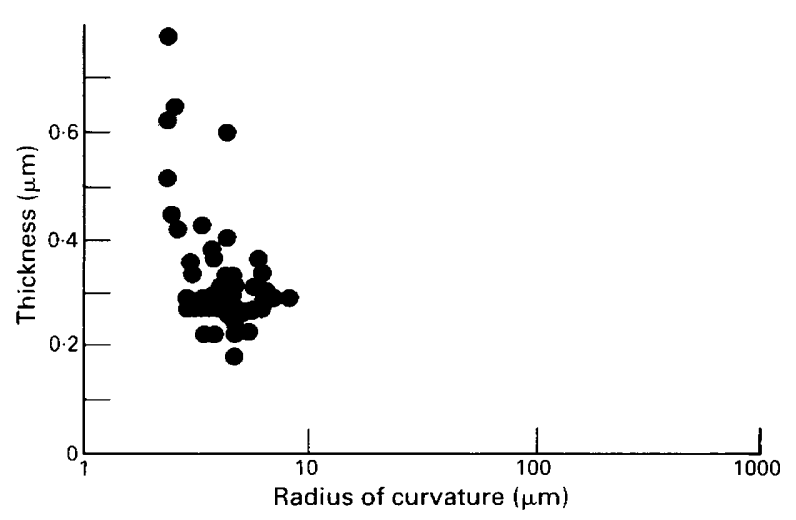

Fig. 5. Distribution of radii of curvature of sections of guinea-pig sperm heads, transverse to the mid-line, as a function of nuclear thickness. Curvature in these sections shows little variation and is limited to $5-10 \mu \mathrm{m}$.

tends to zero, this oblique hypothetical circle, and the perpendicular one from which it derives, will coincide, i.e. the sagitta tends to a minimum. The calculated radius of curvature of the oblique hypothetical circle will be smaller than that of the perpendicular one (because the sagitta is larger for a constant chord length), and therefore provides a measure of the obliqueness of the section, or degree of tilt along the major axis. At the same time, it is possible to identify those sections in a sperm population closest to perpendicularity (i.e. with zero tilt) by measuring nuclear thickness, since this value will also tend to a minimum as obliqueness of the section to the perpendicular disappears. A plot of the hypothetical radius of curvature versus nuclear thickness should, therefore, give a curve which stops at the minimum thickness and maximum radius of curvature.

Using the same principles, the curvature of the sperm head in transverse section (i.e. parallel to the minor axis of the head) can be estimated by taking advantage of the acrosomal cusp. The method is only suitable for spermatozoa with intact acrosomes, i.e. before the acrosome reaction has taken place, and only in those species with marked cusps. This effectively restricts analysis to guinea-pig spermatozoa amongst the common mammalian species. Since scanning electron microscopy and Nomarski light microscopy show the cusp in guinea-pig spermatozoa to be symmetrical (unpublished observations), a transverse section can be identified in transmission electron micrographs when the two wings of the acrosomal cusp are identical in size and shape. As with the head-to-tail sections, these transverse sections will be distributed around a rotational axis, and again, the curve of the hypothetical radius of curvature versus nuclear thickness should terminate at the point where nuclear thickness is a minimum, and give the range for the true radii of curvature.

The results are shown in Figs 5-8. The plot of radius of curvature versus nuclear thickness for transverse sections of guinea-pig sperm nuclei (Fig. 5) is the one which most closely conforms to expectations. From this plot one can conclude that the distribution of radii of curvature lies within 


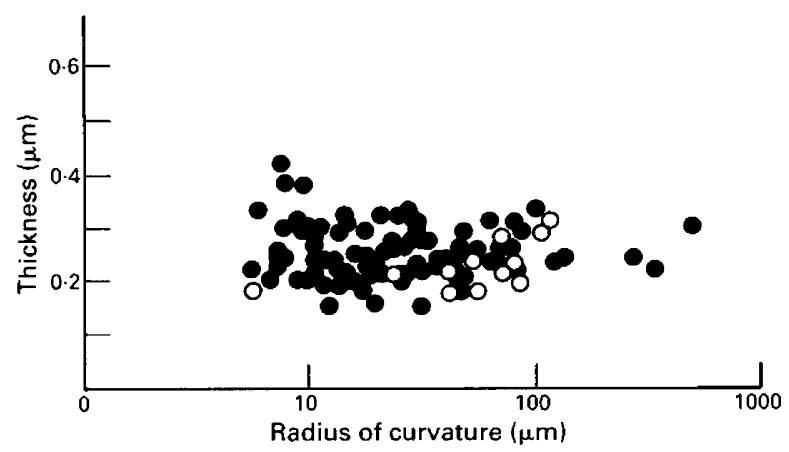

Fig. 6. Distribution of curvature of head-to-tail sections of guinea-pig sperm heads with intact acrosomes, as a function of nuclear thickness. Closed circles indicate spermatozoa with acrosomes lying on the convex face, open circles those with acrosomes lying on the concave face.

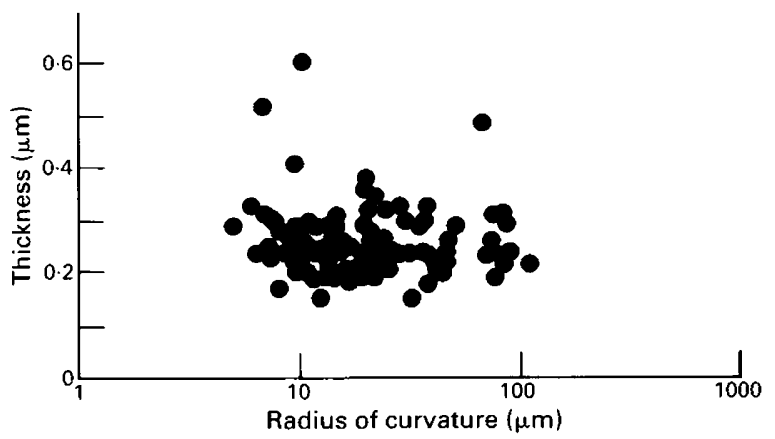

Fig. 7. Distribution of curvature of head-to-tail sections of guinea-pig sperm heads, after the acrosome reaction, as a function of nuclear thickness. The distribution of radii of curvatures has not altered significantly from that in Fig. 6.

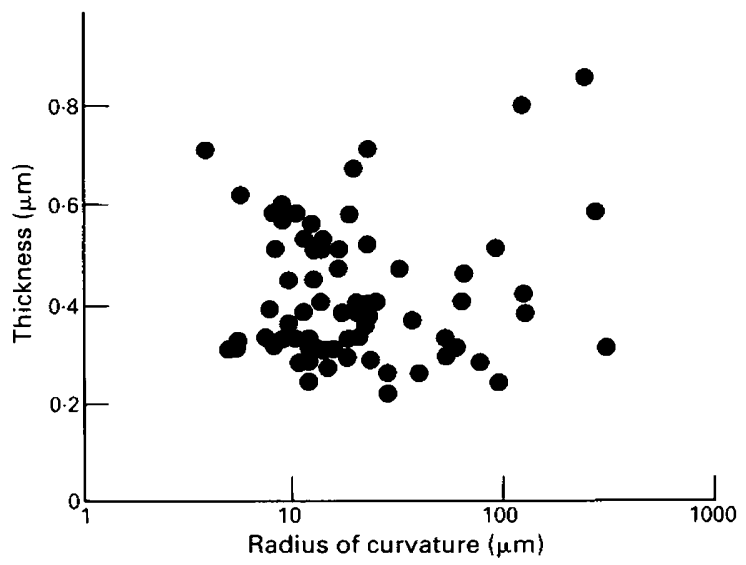

Fig. 8. Distribution of curvature of head-to-tail sections of rabbit sperm heads as a function of nuclear thickness. 

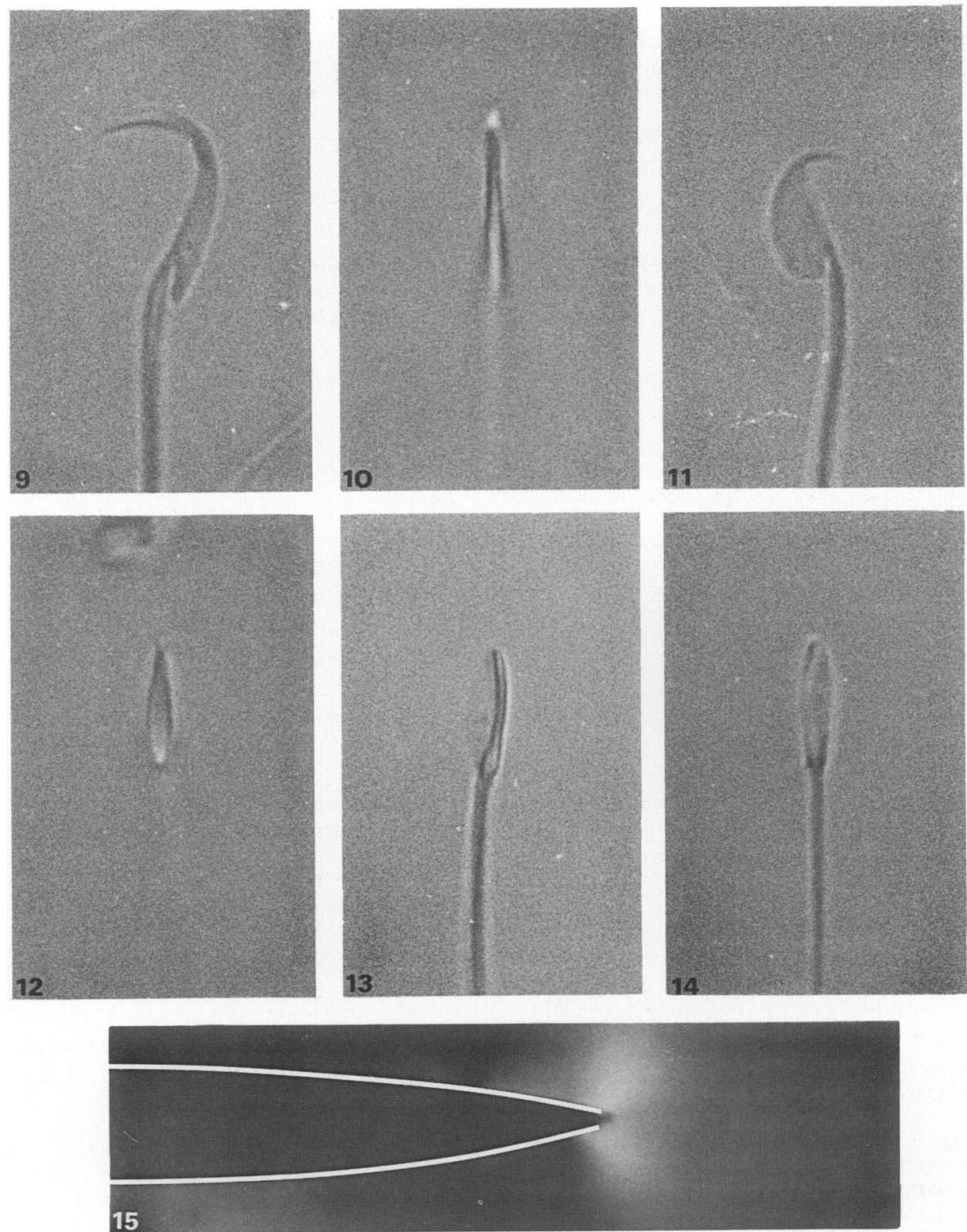
fairly narrow limits, about 3-10 $\mu \mathrm{m}$, and that of nuclear thickness $0 \cdot 15-0 \cdot 30 \mu \mathrm{m}$. Figures $6-8$ show a very much wider spread of curvature for the minimum nuclear thickness. To some extent, this may reflect a sampling error due to the variation in position at which the head is attached to the tail, but the major cause is likely to be simple variation in head shape. The principal criticism which has to be countered at this stage is that this variation is not a natural phenomenon but is, instead, an artefact of fixation. To the argument that spermatozoa are inherently unlikely to show variation, one can point to the variation in the point of attachment of tail to head (Fawcett, 1968), a variation that cannot be attributed to fixation. To counter the second criticism, that curvature is generated by fixation, spermatozoa were examined by Nomarski light microscopy without prior fixation. Although it is not possible to measure the radius of curvature with any accuracy, it is possible to establish qualitatively that the nucleus is normally curved (unpublished observations). Figures $6 \&$ 7 show that the curvature of head-to-tail sections of guinea-pig spermatozoa is nearly always with the convex surface towards the acrosome, and that the distribution of radii does not alter when spermatozoa undergo the acrosome reaction. This suggests that the acrosome is not important in determining the curvature of the nucleus. Figures 6 and 7 also show that, for guinea-pig spermatozoa in head-to-tail section, the nucleus is thicker on average at the point of measurement than in transverse section, and that it lies, in head-to-tail sections, somewhere between about $0 \cdot 15$ and $0.35 \mu \mathrm{m}$; radii of curvature lie, for the most part, between 7 and $100 \mu \mathrm{m}$. On the other hand, the plot of radius of curvature versus nuclear thickness for rabbit spermatozoa (Fig. 8) shows a much greater scatter of points than the equivalent plot for guinea-pig spermatozoa. This in turn, suggests that the distribution of values for nuclear thickness is greater, as well as the actual values or thickness being physically larger $(0 \cdot 2-0 \cdot 4 \mu \mathrm{m})$.

This method of analysis is inapplicable to mouse and rat spermatozoa because their nuclei are asymmetric and lie to one side of any axis determined by the tail (Figs $9 \& 11$ ). Thus, although their nuclei may be considered as approximately flat and lying in a plane, longitudinal rotation about any axis derived from the tail produces no sections perpendicular to this plane which actually pass through the nucleus. Although it is possible to recognize, in light micrographs, a suitable rotational axis parallel to the tail which would pass through the nucleus, it is impossible to establish this axis in electron micrographs. The solution to the problem of establishing curvature lies in examining rat and mouse spermatozoa by light microscopy and utilizing the ability of Nomarski optics to provide optical sectioning. There is still a problem of interpretation caused by the asymmetry of the nucleus, however, because nuclei which are viewed side-on but marginally out of the plane of the head will appear slightly curved (a result of the sickle-shaped nature of the heads). Any curved image is therefore ambiguous: it can be attributed either to a flat nucleus being viewed slightly

Figs 9-12. Nomarski light micrographs of rat and mouse spermatozoa en face and in optical section normal to the plane of the head respectively: Figs $9 \& 10$, rat; Figs $11 \& 12$, mouse. $\times 2210$.

Figs 13 \& 14. Nomarski light micrographs of guinea-pig (Fig. 13) and rabbit (Fig. 14) spermatozoa in optical seçtion normal to the plane of the head. The guinea-pig spermatozoon is without its acrosome. Although the envelope of the rabbit spermatozoon is symmetrical about the longitudinal axis, the nucleus can be seen travelling down one side. $\times 2210$.

Fig. 15. Pattern of transmitted light around the anterior tip of a perspex model, approximately $1 \mathrm{~cm}$ long, of a guinea-pig sperm head after an acrosome reaction. The view is normal to the plane of the head. The head was thrust into a $6 \%$ gelatin gel cross-illuminated with polarized, monochromatic light, and viewed with a crossed polarizer. The light source was a sodium lamp. The pattern shows, in a qualitative way, that the region of highest strain in the gel lies in the region in which a sperm-shaped object might be expected to produce the highest stress concentration from a purely thrusting motion, i.e. immediately anterior to the tip. 
obliquely, or to a curved nucleus being viewed side-on across the plane of the head. It is possible, however, to find many examples of flat nuclei, and an example of each is shown in Figs 10 and 12. The only reasonable interpretation is that they represent flat nuclei viewed side-on. Figures 13 and 14 show similar sections of guinea-pig and rabbit spermatozoa respectively. These clearly show the curvature of the nucleus in the spermatozoa of these two species, demonstrating that Nomarski optics will detect curvature if it is present.

Finally, it is possible to use polarized, monochromatic light to demonstrate, in a purely qualitative way, where the stress concentrations lie for an object shaped like a guinea-pig or rabbit spermatozoon pushing through a gel (Fig. 15). Light is transmitted only where the gel has been strained, and this may be inferred to have occurred in response to the highest stress concentrations. These lie at the anterior tip of the model.

\section{Discussion}

The results in this paper show that, in guinea-pig and rabbit spermatozoa, the nucleus, and therefore the underlying shape of the head, is curved in head-to-tail section, and that in guinea-pigs, it is curved in transverse section also. Taking the head-to-tail and transverse data together, they show that guinea-pig sperm nuclei are caps derived from sections of prolate spheroids. It is not possible, using the methodology described in this paper, to show that guinea-pig spermatozoa after the acrosome reaction remain curved in transverse section (it would require the use of Gibbons' (1963) method to establish this), but given that there appear to be no substantial changes in the curvature of head-to-tail sections in spermatozoa undergoing the reaction, it would be reasonable to assume that nuclear shape remains unchanged. The results for rabbit spermatozoa show much greater scatter (Fig. 8). This, in turn, suggests that rabbit nuclei have intrinsically a very much wider distribution of nuclear thickness at the point of measurement than do guinea-pig spermatozoa. Nevertheless, as with guinea-pig spermatozoa, there is no doubt that rabbit sperm nuclei are curved in head-to-tail section.

The shape of these, and other, sperm heads is of interest primarily because it may determine the curvature of the penetration slit made by spermatozoa in the zona pellucida. The origin of this curvature has so far remained unexplained. If it was determined by head shape, one might expect a close correlation between the radii of curvature of sperm heads in head-to-tail section and those of the penetration slits. Unfortunately, the radii of curvature of the penetration slits through the zona pellucida are difficult to measure accurately, partly because in most species there is a zona block to polyspermy which restricts sperm penetration (thereby entailing that each zona only contains one slit), and partly because, unless the slit is en face, it will be subject to foreshortening. The only species in which large numbers of slits have been examined is the rabbit, in which there is no zona block. This allows a number of slits to be seen in a single egg (Dickmann, 1964). Spermatozoa enter the zona tangentially but arrive at the inner face of the zona at an angle between 60 and $90^{\circ}$ to the tangent. Simple trigonometry shows that spermatozoa which arrive at $90^{\circ}$ travel along an arc whose radius of curvature is the thickness of the zona and those which arrive at $60^{\circ}$ travel in an arc whose radius is double the zona thickness. This thickness varies in the rabbit somewhere between 5 and $20 \mu \mathrm{m}$ and, therefore entails radii of curvature for the slits of between 5 and $40 \mu \mathrm{m}$. Figure 8 shows that $78 \%$ of rabbit spermatozoa have nuclei with radii of curvature within those limits. Some of those datum points will represent sections which are substantially oblique to the perpendicular to the plane of the head and, for these points, the true radius of curvature of the head-to-tail section will be larger. However, the evidence from the transverse guinea-pig sections (Fig. 5) indicates that, even for the most oblique, where measured thickness is at a maximum, the calculated radius of curvature is still not less than half its true value for a perpendicular section. This suggests that, in head-to-tail sections, the radii of curvature for points above minimum thickness have been underestimated by no more than a factor of 2 and this is unlikely to alter substantially the percentage of 
spermatozoa with radii between 5 and $40 \mu \mathrm{m}$. In addition, the observation that, in both species examined, there are radii of curvature for the spermatozoa which are so large that the spermatozoa would fail to penetrate the zona if this were the determining factor is consistent with the observation in rabbit eggs that a proportion of spermatozoa fail to reach the zona despite initial penetration (Dickmann, 1964).

Not all spermatozoa penetrate the zona in a curved path, however. In mouse and rat eggs, and possibly human eggs as well, spermatozoa enter the zona at right angles and move forward in a straight line (Austin \& Braden, 1956; Gaddum-Rosse et al., 1984). It is impossible to analyse electron micrograph sections of rat and mouse spermatozoa in the way outlined for guinea-pig and rabbit spermatozoa because the heads of rat and mouse spermatozoa are severely asymmetric, with the projection of the tail passing to one side of the head (Figs $9 \& 11$ ). Their heads can be sectioned optically, however, by Nomarski light microscopy (Figs 10 \& 12). Provided the head is truly normal to the plane of the slide, the heads are straight.

In summary, all the observations are consistent with the proposal that curvature of sperm heads in longitudinal section is a major determinant of the curvature of the penetration slit (with flat heads being a special case in which the radius of curvature is infinitely large). This proposal raises two obvious questions.

Firstly, curvature of the penetration slit is essential for those spermatozoa which attach tangentially to the zona surface if they are ever to meet the egg. However, if the curvature of the slit is determined by the curvature of the head, it follows that spermatozoa must attach concave side down. This, in turn, implies an asymmetry of binding, for which no direct evidence exists. In part, this may be due to the fact that nobody has found it worth looking for. It may also be due to the subtlety of any differences in the concave and convex surfaces. There is, however, at least one documented example of asymmetry, and that is in the spermatozoa of the musk shrew (Cooper \& Bedford, 1976). Further evidence should not be unduly difficult to find if the phenomenon is real.

Secondly, there has to be a reasonable mechanism by which the curvature of the head does actually produce a curved slit. Two mechanisms have been proposed to account for sperm penetration of the zona pellucida and formation of the penetration slit. In one, a zona-lysin is attached to the sperm surface, enabling spermatozoa to digest a path through the zona pellucida (Austin \& Bishop, 1958; Bedford, 1970). Since spermatozoa achieve a significant fraction of their total thickness immediately posterior to the perforatorium, an important site of zona-lysin activity might be expected to be the leading edge of the spermatozoon. The second proposed mechanism of penetration depends on the dispersal of soluble zona-lysin into the zona pellucida, where it attacks bonds of zona glycoproteins exposed by the mechanical uncoiling induced by sperm thrust (Green, 1987). On this proposal, the leading edge of the spermatozoon would be expected to be the site of proteolysis, because that is where maximum uncoiling of zona proteins is taking place; i.e. that is where the stress concentration is highest. On this mechanism the sperm head would be deflected into the space created at the sperm tip, and follow a path which follows approximately the curvature of the head (in a manner similar to an ice-cream scoop moving into ice-cream). Whether the sperm-bound enzyme would produce an identical pattern of penetration is less certain, but if the sperm-bound zona-lysin were present predominantly on the anterior surfaces of the head, a similar pattern of penetration would appear likely. Again, spermatozoa would move into the space created at the anterior tip, and be deflected into that space by the relative absence of enzymic digestion of the zona on the convex surface of the head.

More detailed arguments concerning the mechanics of penetration must await an integration of the known movements of the head (which in mammalian spermatozoa also includes a significant side-to-side movement as well as a substantial turning moment or torque) and a more detailed knowledge of the dimensions of the penetration slit. For example, it is not known at present where spermatozoa exert their maximum stress. For forward thrusts, however, the thrust exerted by the tail on the head can be resolved into normal and tangential components, with the normal 
component exerting stress and the tangential component overcoming any drag. (The principles involved were clearly outlined for somewhat different circumstances by Gray (1953).) At the anterior tip, normal components lie predominantly longitudinally with respect to the sperm head and exert, over the small area of the perforatorium, the major stress concentration produced by thrust. It is possible to demonstrate this in a qualitative way using a perspex model for the sperm head in a gelatin gel illuminated with monochromatic, polarized light (Fig. 15). This type of approach has been used for many years to establish stress concentrations in models of structures such as dams. What distinguishes the approach here is the use of scaling up, rather than scaling down. The underlying assumption is, of course, that such an approach is valid. This may not be entirely the case since, at the macroscopic level, a gel can be treated as isotropic whereas, at the microscopic level, this assumption begins to break down. Nevertheless, the model is a useful indicator of the area in which bonds are most likely to be placed under tensile load by a sperm head executing a forward thrusting movement. In this, it supports the conclusions arrived at above.

A consideration of the mechanics of penetration is likely to have widespread application. To give but one example: a number of animals have spermatozoa which have helical or screw threadlike heads (Fawcett et al., 1971; Mizuhira et al., 1986). In the case of Rhacophorus, it is known that the spermatozoa rotate in the same sense as the helix, i.e. counterclockwise when viewed forward from the tip of the tail (Mizuhira et al., 1986). These spermatozoa are potentially capable therefore of screwing their way into an egg provided their tail generates sufficient torque, and provided they rotate, as they do in Rhacophorus, in the same direction as the pitch of the helix. The advantage of this adaptation from the spermatozoon's point of view may be a greater forward thrust, since it is known experimentally that rotational forces can be at least an order of magnitude greater than direct thrust (Baltz \& Cone, 1986). Although the forward thrust can be no more than $50 \%$ of the rotational force and possibly less, depending on the pitch of the helix, forward thrust from screwing could, on the figures of Baltz \& Cone (1986) still be as much as a factor of 5 greater than that from direct flagellar propulsion. The torques in those species with helical heads may, of course, be much larger.

This work was supported by the Medical Research Council of New Zealand.

\section{References}

Austin, C.R. \& Bishop, M.W.H. (1958) Role of the rodent acrosome and perforatorium in fertilization. Proc. R. Soc. B. 149, 241-248.

Austin, C.R. \& Braden, A.W.H. (1956) Early reactions of the rodent egg to spermatozoon penetration. J. exp. Biol. 33, 358-365.

Baltz, J. \& Cone, R.A. (1986) Flagellar torque on the head determines the force needed to tether a sperm. Biophys. J. 49, 78a.

Bedford, J.M. (1970) Sperm capacitation and fertilization in mammals. Biol. Reprod., Suppl. 2, $128-158$.

Bleil, J.D. \& Wassarman, P.M. (1980) Structure and function of the zona pellucida: identification and characterization of the proteins of the mouse oocyte's zona pellucida. Devl Biol. 76, 185-202.

Cooper, G.W. \& Bedford, J.M. (1976) Asymmetry of spermiation and sperm surface charge patterns over the giant acrosome of the musk shrew Suncus murinus. J. Cell Biol. 69, 415-428.
Dickmann, Z. (1964) The passage of spermatozoa through and into the zona pellucida of the rabbit egg. J. exp. Biol. 41, 177-182.

Dickmann, Z. \& Dziuk, P.J. (1964) Sperm penetration of the zona pellucida of the pig egg. J. exp. Biol. 41, 603-608.

Dietl, J. \& Czuppon, A.B. (1984) Ultrastructural studies of the porcine zona pellucida during the solubilization process by Li-3,5-diiodosalicylate. Gamete Res. 9, 45-54.

Dziuk, P.J. \& Dickmann, Z. (1965) Sperm penetration through the zona pellucida of the sheep egg. J. exp. Zool. 158, 237-239.

Fawcett, D.W. (1965) The anatomy of the mammalian spermatazoon with particular reference to the guinea-pig. Z. Zellforsch. mikrosk. Anat. 67, 279-296.

Fawcett, D.W. (1968) The topographical relationship between the plane of the central pair of flagellar fibrils and the transverse axis of the head in guineapig spermatozoa. J. Cell Sci. 3, 187-198. 
Fawcett, D.W., Anderson, W.A. \& Phillips, D.M. (1971) Morphogenetic factors influencing the shape of the sperm head. Devl Biol. 26, 220-251.

Friend, D.S. \& Fawcett, D.W. (1974) Membrane differentiations in freeze-fractured mammalian sperm. J. Cell biol. 63, 641-664.

Gaddum-Rosse, P., Blandau, R.J., Langley, L.B. \& Battaglia, D.E. (1984) In vitro fertilization in the rat: observations on living eggs. Fert. Steril. 42, 285-292.

Gibbons, I.R. (1963) A method for obtaining serial sections of known orientation from single spermatozoa. J. Cell Biol. 16, 626-629.

Gray, J. (1953) Undulatory movement. Quart. J. microsc. Sci., N.S. 94, 551-578.

Green, D.P.L. (1978) The induction of the acrosome reaction in guinea-pig sperm by the divalent metal cation ionophore A23187. J. Cell Sci. 32, 137-151.

Green, D.P.L. (1987) Mammalian sperm cannot penetrate the zona pellucida solely by force. Expl Cell Res. 169, 31-38.
Greve, J.M. \& Wassarman, P.M. (1985) Mouse egg extracellular coat is a matrix of interconnected filaments possessing a structural repeat. J. molec. Biol. 181, 253-264.

Mizuhira, V., Futaesaku, Y., Ono, M., Ueno, M., Yokofujita, J. \& Oka, T. (1986) The fine structure of the spermatozoa of two species of Rhacophorus (arboreus, schlegelii). J. Ultrastruct. molec. Struct. Res. 96, 41-53.

Yanagimachi, R. (1966) Time and process of sperm penetration into the hamster ova in vivo and in vitro. J. Reprod. Fert. 11, 359-370.

Yang, W.H., Liu, L.L., Wang, J.R. \& Chang, M.C. (1972) Sperm penetration through zona pellucida and peri-vitelline space in the hamster. J. exp. Zool. 179, 191-206.

Received 22 September 1987 